\title{
Comparison of Intensity-based B-splines and Point-to-Pixel Tracking Techniques for Motion Reduction in Optical Mapping Experiments
}

\author{
Jaime Yagüe-Mayans $^{1}$, Antonio Guill ${ }^{1,2}$, Manolo Zarzoso ${ }^{2,3}$, Antonio Cebrián ${ }^{1}$, Francisco J Chorro ${ }^{2,3}$, \\ José Millet ${ }^{1,2}$, Conrado J Calvo*1, 2 \\ ${ }^{1}$ BioITACA, Dpt. Ingeniería Electrónica, Universitat Politécnica de València, València, Spain \\ ${ }^{2}$ Grupo de Electrofisiología y Bioingeniería, IIS INCLIVA-Hospital Clínico, Universitat de València, Spain \\ ${ }^{3}$ Departamentos Fisioterápia, Fisiología y Medicina, Universitat de València, Spain
}

\begin{abstract}
Suppression of motion artifacts (MA) in cardiac optical mapping usually requires uncoupling of cardiac contraction by restriction techniques, which are known to have important effects on cardiac physiology deteriorating the quality of acquisitions and their interpretation. In this study, we propose to assess the performance of two independent intensity-based postprocessing strategies to minimize MAs during registration. A point-to-pixel block-matching classical similarity-based tracking with displacement interpolation is compared to a well-known non-rigid registration algorithm where the deformation field is obtained using cubic splines. Both strategies were tested on synthetic and real optical mapping sequences, and further compared based on their tracking accuracy and ability to maintain activation and repolarization information of the reconstructed action potentials in wide areas of the epicardial surface. The algorithms proved to be accurate tracking frame-to-frame axial geometrical displacements. Yet, special care must be taken to preserve upstroke information intact.
\end{abstract}

\section{Introduction}

In the last two decades, cardiac optical mapping (OM) has become a valuable tool for the study of cardiac physiology to understand the mechanisms behind complex arrhythmias and conduction disorders $[1,2]$. The high spatial and temporal resolution achieved by this technique, the possibility of studying several physiological parameters simultaneously, as well as, allowing to study the transient events during defibrillation shocks; make OM very attractive as compared to traditional electrical mapping strategies.

However, inherent heart motion and contraction during acquisition gives rise to motion artifacts (MAs), which deteriorate the quality of the sequences and hinder their analysis and interpretation. MAs manifest both as phase artifacts due to uncorrected registration and as a modulation of the signal of interest caused by different mechanisms including heterogeneities in dye distribution $[3,4]$ or excitation light intensity [5], as well as changes in tissue volume [6,7].

To date, either motion restriction techniques such as mechanical restraint or chemical uncouplers are used to avoid superimposed MAs, however those techniques may alter cardiac physiology, whose alterations are still a matter of debate [8]. Although many alternatives based on post-processing techniques have been proposed to address this issue [3-6] none of them has become widely accepted. Among previously proposed algorithms, image registration techniques $[3,4,5,6]$ have generated a great interest, since only these methods account for phase errors. However, comprehensive evaluation of these techniques may be bias due to heterogeneous results and technical setups. In this work we aim to assess the performance of two independent image registration algorithms, in the same controlled acquisitions and synthetic sequences. We test two post-processing pipelines: a point-to-pixel similarity tracking approach (PPBM) [9] and a classical non-rigid registration algorithm where the deformation field is obtained using cubic splines (DFCS) [4].

\section{Methodological approaches}

\subsection{Sequences database}

We defined a database including a synthetic sequence (phantom) and real OM sequences $(\mathrm{n}=5)$. We designed the phantom using the following procedure. First, the initial frame was generated from a real acquisition and each 'active' pixel was assigned to a synthetic signal mimicking activation and repolarization phases of action potentials. Second, relative delays on pixel signals were introduced for different pixels based upon prior knowledge on activation from numerical models when stimulating from the upper left corner to simulate propagation. Third, prior knowledge on intrinsic heart 
motion allowed us to define known non-rigid spatial transformations to each frame. Finally, we post-process the synthetic sequence by cropping and masking the area of interest (71x71 pix., 350 fr.). The synthetic sequence simulates 3 cardiac cycles with superimposed realistic action potentials and motion.

We also considered real OM acquisitions obtained from Langendorff-perfused isolated rabbit heart preparations where homogenous dye distribution was ensured. Acquisitions were obtained using custom-built illumination system with a near-infrared emission dye opto-mechanical configuration. We obtained sequences with $128 \times 128$ pixels and frame-rates of $0.5 \mathrm{KHz}$ at fullframe. Acquisition was synchronized with light excitation as previously done [9] and controlled using Matlab and Labview. Sequences were obtained during sinus rhythm after 15 minutes of stable beating and after the infusion of the uncoupler blebbistatin (BB). Segments of 2 seconds duration were used for analysis purposes.

\subsection{Image registration using point-to- pixel tracking and interpolation techniques (PPBM)}

The point-to-pixel with block-matching strategy comprises mainly of the following steps implemented in Matlab (Matlab 2013a, The Mathworks, Inc. Natick):

1. Tracking of semi-automatically selected reference points. Sub-pixel resolution was estimated using spatial sub-sampling and bilinear interpolation. A pre-defined grid was defined with 30 pixel spacing with automatic rejection of points outside or near the heart boundaries. Automatically selected key-points were tracked through the sequence using a block-matching algorithm based on the classical similarity normalized cross-correlation coefficient. We defined a 27x27px sized reference blocks for each point and the maximum displacement between consecutive frames was limited to 6px in each direction. Detected trajectories were then returned fitted to the original grid and post-processed.

2. Piecewise bilinear interpolation of detected displacements. We defined a triangular-element grid using Delaunay-triangulation using the reference points as nodes. Displacements at those nodes were bilinearly interpolated to estimate motion of all points within the meshed region of interest.

3. Image registration. Image registration was achieved by applying the inverse of the estimated spatial transformation. Since both detected and interpolated displacements had sub-pixel accuracy we used distance weighted averages of the four-nearest neighbours to the estimated position.

\subsection{Image registration using cubic b-}

\section{splines based deformation fields (DFCS)}

A strategy based on using cubic B-splines deformation fields for motion compensation was previously described by Westergaard P. et al [4]. In this case, the spatial transformation is approximated optimizing the deformation field in equation (1),

$$
M_{i j}=\sum_{k=0}^{n} \sum_{l=0}^{n} N_{k, d} *\left(\frac{i}{p 0-1}\right) * N_{l, d} *\left(\frac{j}{p 1-1}\right) * B_{k l}
$$

where $N(u)$ are the b-splines basis functions and $B k l$ are the control points. The value $n$ defines the number of such points in each axis and d determines the degree of the basis function. The positions of control points depend upon the selected basis functions, in our case cubic bsplines.

This deformation field is iteratively optimized, moving a control point at a time, by the maximization of a global similarity measure. In [4], they used normalized mutual information for this purpose, but we used correlation ratio instead in order to maintain the same methodological intensity-based similarity metric. A two-pass registration approach was used as suggested in [4]. First, the method was applied using 16 control points in each axis with a step size (Ss) of $0.5 \mathrm{px}$, convergence limit $(\mathrm{Cl})$ set at 0.1 and the maximum iterations limited to 100 . In the second pass 32 control points in each axis were used instead (Ss $0.25 \mathrm{px}, \mathrm{Cl} 0.01$, It 100). The first step aimed to reduce coarse motion, while the second one sought for refining tracking accuracy. This method was applied using MIPAV (Medical Image Processing, Analysis and Visualization, http://mipav.cit.nih.gov), a publically available Java application provided by National Institutes of Health, Centre for Information Technology [4].

\subsection{Signal processing considerations and performance evaluation}

Signals obtained from real acquisitions were preprocessed in time, inverted and normalized, and space, each frame was spatially filtered using a Gaussian 3x3smoothing kernel. Motion tracking accuracy was evaluated in the synthetic sequences. Motion compensation was quantified by comparing the total directional displacement (TDD) reduction derived from detected trajectory loops before and after compensation with each methodology. Evaluation of AP morphological changes and quantification of the spatial dispersion on cardiac electrophysiology after reconstruction were evaluated through measures of action potential duration at $80 \%$ of repolarization $\left(\mathrm{APD}_{80 \%}\right.$ ) and activation times though upstroke depolarization velocity using the maximum derivative of the AP to generate isochronal maps before and after compensation. Acquisitions in 
presence of $\mathrm{BB}$ were used to compare the spatial dispersion in real $\mathrm{OM}$ sequences. All analyses were performed on Matlab2013a using custom made tools.

\section{Results}

Our results show that motion was substantially suppressed at automatically selected initial grid keypoints (Table 1, Figure 1). Trajectory loops were obtained using PPBM tracking with real and synthetic sequences. This method was validated with the synthetic sequence, where RMSE between real and detected trajectories was in the sub-pixel range $(0.42 \mathrm{px})$. Total directional displacement (TDD) derived from detected trajectories was reduced by a $72.8 \%$ in average with PPBM and by a 73.9\% with DFCS (Table 1). DFCS reduced the mechanical deformation further also with the phantom sequence (68.87 vs $56.46 \%)$.

Table 1. Summary of numeric results obtained with both methods in real and synthetic sequences.

\begin{tabular}{lccc}
\hline & $\begin{array}{c}\text { TDD } \\
(\mathbf{p x})\end{array}$ & $\begin{array}{c}\text { APD } \\
(\mathbf{m s})\end{array}$ & $\begin{array}{c}\mathbf{V}_{\mathbf{d}} \\
(\boldsymbol{\partial} \mathbf{V m} / \partial \mathbf{t})\end{array}$ \\
\hline \multicolumn{3}{c}{ REAL SEQUENCES } \\
\hline Original & $3.56 \pm 0.62$ & $120.5 \pm 27.42$ & $0.28 \pm 0.04$ \\
PPBM & $0.97 \pm 0.26$ & $109.53 \pm 3.94$ & $0.30 \pm 0.03$ \\
DFCS & $0.93 \pm 0.52$ & $109.89 \pm 2.69$ & $0.29 \pm 0.02$ \\
Uncoupled & $0.37 \pm 0.24$ & $128.84 \pm 2.25$ & $0.31 \pm 0.04$ \\
\hline \multicolumn{4}{c}{ PHANTOM } \\
\hline Original & $3.33 \pm 1.28$ & $212.51 \pm 99.21$ & $0.31 \pm 0.06$ \\
PPBM & $1.45 \pm 0.99$ & $159.06 \pm 27.61$ & $0.34 \pm 0.06$ \\
DFCS & $1.04 \pm 0.75$ & $156.88 \pm 12.75$ & $0.26 \pm 0.08$ \\
Real & - & 150 & 0.39 \\
\hline
\end{tabular}

The greater the spatial motion reduction, the lesser MAs were superimposed in early phases of the AP. Additionally, APD $>75 \%$ showed lesser dispersion as it was measure more accurately. Figure 2, show how $\mathrm{APD}_{80}$ measures are more evenly distributed with lesser spatial dispersion after correction, except for residual MAs found in small areas near the boundaries. These regions would be rejected during post-processing for spatial quantification. Overall dispersion on $\mathrm{APD}_{80}$ measures in the resulting areas was similar to the dispersion under the effects of the uncoupler BB, and considerably lower than that observed in the original sequences. Also, measures obtained with both methods were very similar across all sequences, with an average difference of $0.61 \pm 0.58 \mathrm{~ms}$. APD dispersion was also considerably reduced in the phantom sequence (Table 1).

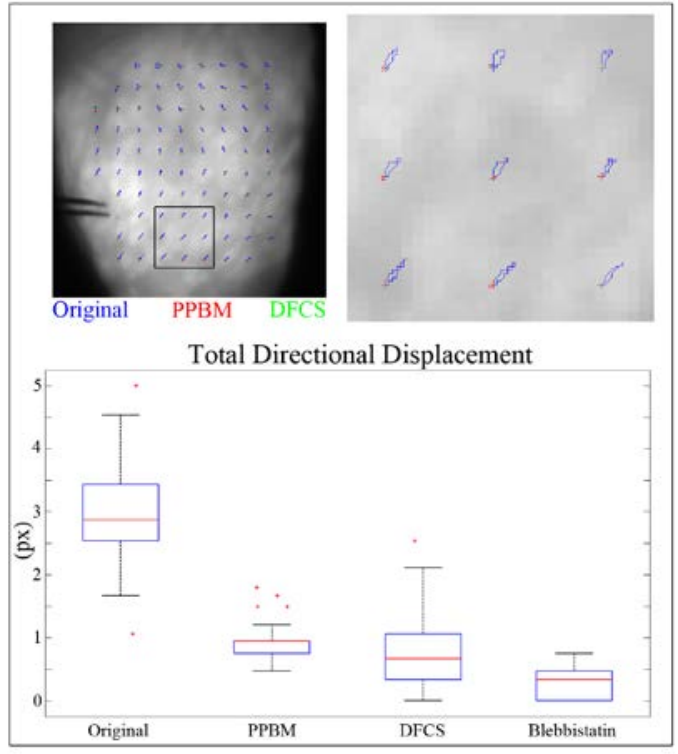

Figure 1. Top. Trajectory loops at pre-selected reference key-points and zoom-in region. Bottom. Boxplot showing total directional displacement (TDD) measures.

The effect of the motion compensation strategies on AP morphology can be observed in figure 3 . Superimposed signals from a $5 \times 5$ neighborhood at locations highlighted in Figure 2 are shown. Variability is reduced even in the boundary areas with residual MAs.

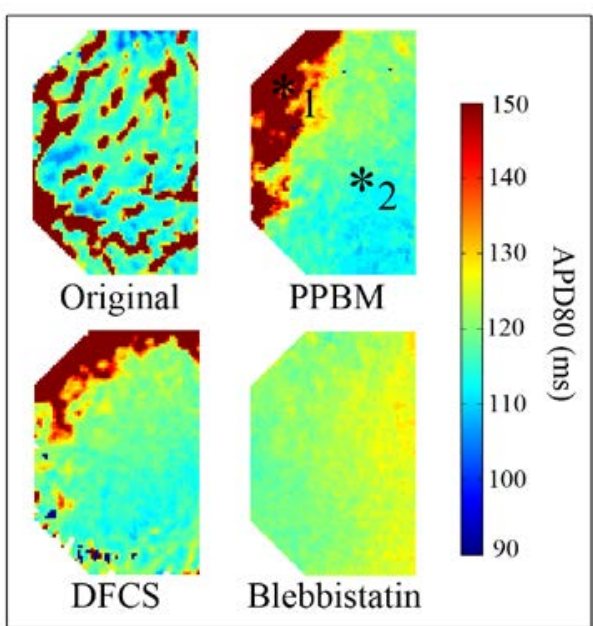

Figure 2. APD80 maps showing heterogeneity reduction in these measures due to motion compensation.

However, $V_{d}$ measurements (Table 1) point out that both methods, but especially DFCS, distorted the AP upstroke, underestimating depolarization measurements. As a result, activation isochronal maps do not reliable represent the activation process, which are specially altered when using the DFCS compensation algorithm. 


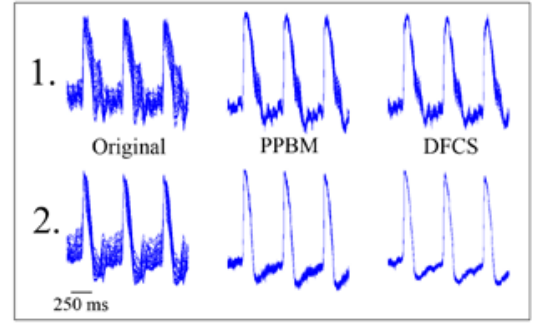

Figure 3. Single pixel transients from $5 \times 5$ neighborhoods reconstructed at specified locations in Figure 2. Loc\#1 represents a region with residual MAs. Loc\#2 represent a wellcompensated area.

\section{Discussion and conclusions}

In this study two image registration algorithms for MA suppression in OM sequences have been compared. Motion was greatly reduced due to effective geometrical displacement tracking, minimizing MAs in reconstructed OM-APs in large areas of the heart wall. This observation was evident in the phantom sequence where we achieved sub-pixel accuracy using both strategies. These results highlight the power of intensity-based similarity measures for motion tracking in this application.

Reconstructed AP morphologies after correction showed to fairly recover the AP transients across the heart wall minimizing the artifacts also during the rest phases. Repolarization was accurately recovered, which was further supported by the reduction in $\mathrm{APD}_{80 \%}$ dispersion similar to that obtained with chemical uncouplers. Altogether, spatial averaged measures of AP duration were in accordance with the true values in the phantom sequence and, in between, of the mean values for original vs uncoupled sequences, which may be also partially explained by the effects of BB on cardiac physiology.

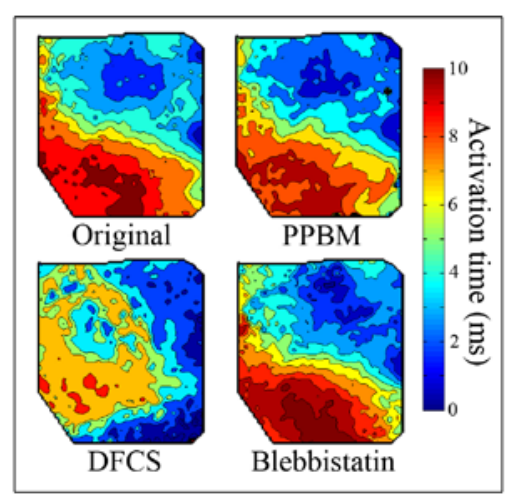

Figure 4. Isochronal activation maps before and after correction with both methods and after the effect of BB.

However, residual MAs are present despite the algorithm employed. These areas, corresponding to the most susceptible boundaries, may be further reduced by further homogenizing excitation and/or using additional gentle restriction approaches. Yet, the main limitation of the methods presented here is the lack of ability to preserve the upstroke information intact due to excessive spatial smoothing. Increasing the temporal resolution during acquisition, and adjusting the spatial displacement process could minimize this unwanted smoothing effects.

In conclusion, intensity based methods are useful for MA correction via registration due to its accuracy tracking frame-to-frame geometrical displacements in large areas of the heart. Yet, special care must be taken to preserve upstroke information intact.

\section{References}

[1] Igor R. Efimov, Vladimir P Nikolsky, Guy Salama. Optical Imaging of the heart. Circulation Research 2004; 95:21 - 33.

[2] Herron TJ, Lee P and Jalife J. Optical Imaging of Voltage and Calcium in Cardiac Cells \& Tissues. Circulation Research 2012; 110:609-623.

[3] Elliot B. Bourgeois, Andrew D. Bachtel, Jian Huang, Gregory P. Walcott, and Jack M. Rogersa. Simultaneous optical mapping of transmembrane potential and wall motion in isolated, perfused whole hearts. Journal of Biomedical Optics September 2011; 16(9) 096020.

[4] Westergaard P., Umapathy K., Masse, S. Sevapstisidis, E. Asta, J. Farid, T., ... \& Nanthakumar, K. Non-linear image registration for correction of motion artifacts during optical imaging of human hearts. Electrical and Computer Engineering, 2008. CCECE 2008. Canadian Conference on (pp. 001729-001732). IEEE.

[5] Inagaki, M., Hidaka, I., Aiba, T., Tatewaki, T., Sunagawa, K., \& Sugimachi, M. (2004, September). High resolution optical mapping of cardiac action potentials in freely beating rabbit hearts. In Engineering in Medicine and Biology Society, 2004. IEMBS'04. 26th Annual International Conference of the IEEE (Vol. 2, pp. 3578-3580). IEEE.

[6] Khwaounjoo P., Rutherford S. L., Svrcek M., LeGrice I. J., Trew M. L., Smaill B. H. (2015). Image-Based Motion Correction for Optical Mapping of Cardiac Electrical Activity. Annals of biomedical engineering, 43(5), 1235-1246.

[7] Martin J. Bishop, David J. Gavaghan, Natalia A. Trayanova, Blanca Rodriguez, Photon scattering effects in optical mapping of propagation and arrhythmogenesis in the heart, Journal of Electrocardiology, 40(6), November-December 2007, Pages S75S80.

[8] Brines L, Such-Miquel L, Gallego D, Trapero I, Zarzoso M, Soler C, Pelechano F, Cánoves J, Alerola A, Such L, Chorro FJ. Modifications of mechanoelectric feedback induced by 2,3butanedione monoxime and Blebbistatin in Langedorff-perfused rabbit hearts. Acta Physiol. 2012; 206(1):29-41.

[9] Lee P, Bollensdorff C, Quinn A, et al Single-sensor system for spatially resolved, continuous and multiparametric optical mapping of cardiac tissue Heart Rhythm 8,9:1482-1491

Address for correspondence.

Conrado J. Calvo

BIO-ITACA Institute

Universitat Politécnica de València

Edificio 8G, Acceso B $3^{\mathrm{a}}$ planta

Camino de Vera s/n

46022, Valencia, España

conradca@itaca.upv.es 